\title{
Transatlantica
}

Revue d'études américaines. American Studies Journal

\section{Blacking Up : Une histoire du rock au prisme du blackface}

\section{Keivan Djavadzadeh}

\section{(2) OpenEdition}

1 Journals

\section{Édition électronique}

URL : https://journals.openedition.org/transatlantica/6553

DOI : $10.4000 /$ transatlantica.6553

ISSN : 1765-2766

Éditeur

Association française d'Etudes Américaines (AFEA)

Édition imprimée

Date de publication : 31 décembre 2013

\section{Référence électronique}

Keivan Djavadzadeh, «Blacking Up : Une histoire du rock au prisme du blackface», Transatlantica [En ligne], 2 | 2013, mis en ligne le 03 mai 2014, consulté le 01 février 2023. URL : http:// journals.openedition.org/transatlantica/6553; DOI : https://doi.org/10.4000/transatlantica.6553

Ce document a été généré automatiquement le 1 février 2023.

\section{c) (i) $९$}

Creative Commons - Attribution - Pas d'Utilisation Commerciale - Pas de Modification 4.0 International - CC BY-NC-ND 4.0

https://creativecommons.org/licenses/by-nc-nd/4.0/ 


\title{
Blacking Up : Une histoire du rock au prisme du blackface
}

\author{
Keivan Djavadzadeh
}

Now my brodder niggars, Eh j'vous parle mes

frères nègres

I do not think it right, Je trouve que c'est pas

bien,

Dat you should laugh at dem De se moquer de Who happen to be white. Ceux qui sont blancs.

Kase it dar misfortune C'est pas leur faute And dey'd spend ebery dollar, Et ils donneraient

leur fortune

If dey only could be Pour être un jour Gentlemen ob colour Des monsieurs de couleur ${ }^{1}$. Paroles de « Jim Crow », classique du répertoire ménestrel blackface

«Blacking Up $»^{2}$. Se faire « Noir ». Le spectacle de ménestrel blackface désigne une forme de théâtre populaire apparue dans la première moitié du XIX ${ }^{e}$ siècle aux ÉtatsUnis qui voyait des comédiens, exclusivement blancs dans un premier temps, se maquiller le visage à l'aide d'un bouchon de liège brûlé ${ }^{3}$. Produit des «politiques raciales de la culture des années ayant précédé la Guerre Civile » ${ }^{4}$ (Lott, 1993, 15), le spectacle ménestrel blackface aura marqué de son empreinte la culture populaire américaine et il n'est sans doute pas exagéré d'affirmer, comme le fait William Lhamon, qu'il s'agit du " premier phénomène de culture de masse atlantique » (Lhamon, 2008, 96). L'attrait qu'ont exercé les spectacles de ménestrels blackface durant plus d'un siècle explique pourquoi la disparition du visage noirci au charbon dans les années 1950 n'a pas mis fin à la réalité que recouvrait cette pratique, dont on retrouve des avatars dans la musique populaire moderne (Tosches, 2003; Lhamon, 2008). La dimension univoquement raciste des spectacles de ménestrels blackface a longtemps constitué un lieu commun des recherches sur le sujet avant que des études plus récentes ne complexifient l'analyse du blackface et en montrent l'ambivalence (Toll, 1977 ; Bean et 
al., 1996 ; Lhamon, 2008). Sans passer sous silence les implications racistes du blackface, Robert Toll, Nick Tosches, William Lhamon et d'autres ont mis au jour que derrière les stéréotypes avilissants, des processus d'identification à certains traits de l'identité noire et d'appropriation de la culture noire se jouaient également en coulisse. L'identité blanche ayant été définie aux États-Unis selon une conception de classe ${ }^{5}$, les Blancs les plus pauvres, ceux qui brouillaient la configuration raciale du pays par leur simple condition sociale, ne pouvaient se retrouver dans la blanchité hégémonique. Ni Blancs ni Noirs, ils ont fait le choix de se façonner une identité hybride en empruntant des signes «noirs ». Cependant, et ce sera mon point central dans cet article, c'est une certaine idée de la blackness ${ }^{6}$ qui a été intégrée à la culture dominante américaine, bien plus que les Noirs eux-mêmes. Car aussi convaincante que soit la démonstration d'auteurs comme Tosches ou Lhamon, ces derniers ne s'interrogent pas sur les processus de déformation et surtout de dépossession à l'œuvre. Et pour cause, Lhamon estime que « les gestes peuvent s'acheter et se vendre mais non se posséder » (Lhamon, 2008,12 ). Pourtant, l'exploitation commerciale qui a été faite (et qui continue d'être faite) de la blackness par les industries culturelles n'a que peu profité aux Noirs mais bien plus aux Blancs qui ont performé certains traits considérés comme caractéristiques de l'identité noire et ont contribué à « dé-racialiser » la blackness. Cet article ne se donne donc pas pour but de démasquer une musique noire véritable, " authentique ", qui aurait été récupérée par des Blancs. Assurément, le rock est une musique aux origines multiples qui se caractérise par une hybridation de différentes influences, noires comme blanches ${ }^{7}$. Je cherche plutôt à brosser l'histoire du rock à rebrousse-poil (Benjamin, 2000, 432-33) et à comprendre les mécanismes qui ont conduit à l'appropriation de certains traits noirs considérés comme caractéristiques par les industries culturelles blanches tout en évinçant ceux qui étaient les porteurs de ces signes. Je me propose donc d'explorer une généalogie de l'appropriation commerciale de la blackness par les maisons de disques sur la période qui va de la commercialisation du blues (années vingt) à l'appropriation commerciale de cette musique par le marché musical blanc. Je souhaite ainsi montrer comment la subculture $e^{8}$ rock des années cinquante s'est construite, entre autres choses, sur la blackness mais qu'en raison des politiques raciales des maisons de disques, les Noirs n'ont bénéficié que marginalement de l'intérêt croissant pour la musique noire.

2 Je vais commencer cet article en revenant brièvement sur l'histoire des maisons de disques américaines. En retraçant l'histoire qui mène des race records au rhythm and blues, je montrerai que le caractère ségrégué de la musique populaire américaine a constitué un terreau fertile pour que survive le cycle blackface dans l'industrie du disque. Car s'il a longtemps existé deux marchés respectivement et exclusivement destinés à un public blanc ou noir, le marché blanc a quasiment systématiquement offert des reprises (covers) des plus grands tubes du répertoire rhythm and blues. J'affirme que la logique qui présidait derrière cette volonté d'offrir de la musique noire "blanchisée ", gommée de ses "aspérités" raciales, consistait à produire de la blackness mais sans les Noirs. À cet égard, le cas d'Elvis Presley sur lequel je vais m'appuyer est exemplaire. Enfin, je m'intéresserai au moment de "redécouverte" d'artistes noirs américains dans les années soixante en montrant que ces artistes qui se produisaient devant un public blanc ont eux aussi dû revêtir le masque blackface pour servir à leur audience le frisson d'une supposée authenticité raciale (Keil, 1966 ; O'Neal, 1994). 


\section{Politiques raciales des maisons de disques américaines : des race records au rhythm and blues}

«Il y a quatorze millions de Noirs dans notre grand pays et ils achèteront des disques s'ils sont enregistrés par l'un des leurs ${ }^{9}$

Perry Bradford, 1920 (Bradford, 1965, 114).

Le 10 août 1920, Mamie Smith entre dans les studios de Okeh Records et grave «Crazy Blues", qui restera dans l'histoire comme le premier disque de blues jamais enregistré $^{10}$. Les publicités mettent en avant le caractère "racial » de la musique et pavent le chemin pour les publicités de race records qui suivront, avec leurs représentations héritées du blackface ${ }^{11}$.

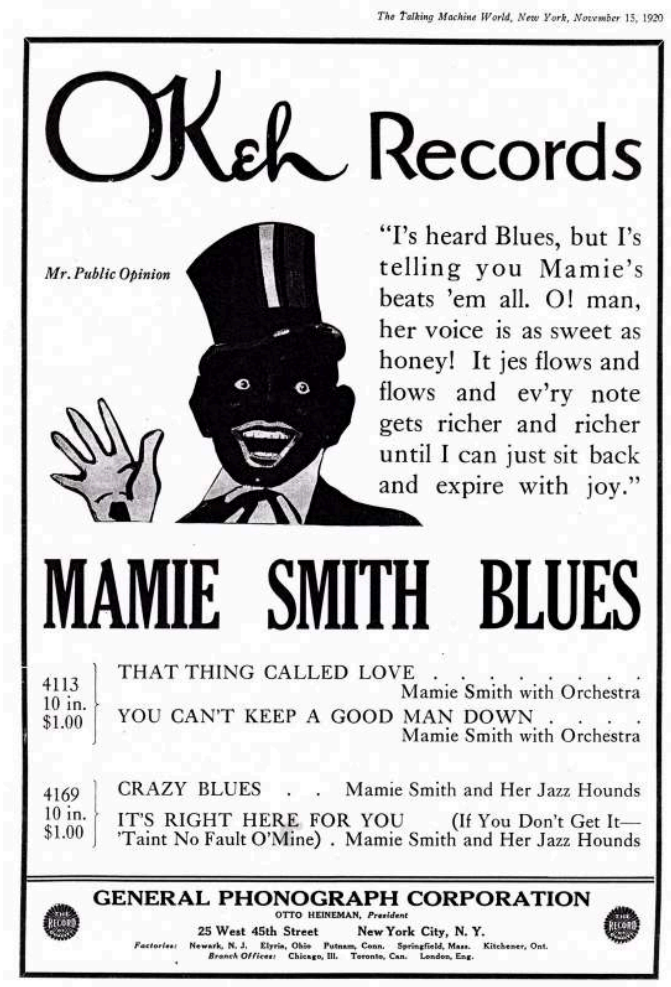

Publicité d'époque Okeh Records pour «Crazy Blues »

On observe ainsi un « M. Opinion Publique » (Mr. Public Opinion) dont seuls les yeux et la bouche ressortent du visage noir charbonneux. Vêtu d'un haut de forme, d'un costume et de gants blancs tel Zip Coon ${ }^{12}$, il vante en " petit nègre " - comme si le langage du blackface était le langage vernaculaire des Africains-Américains - les mérites du disque : «Je entendu blues, mais je dis vous Mamie c'la meilleure ${ }^{13}$. 


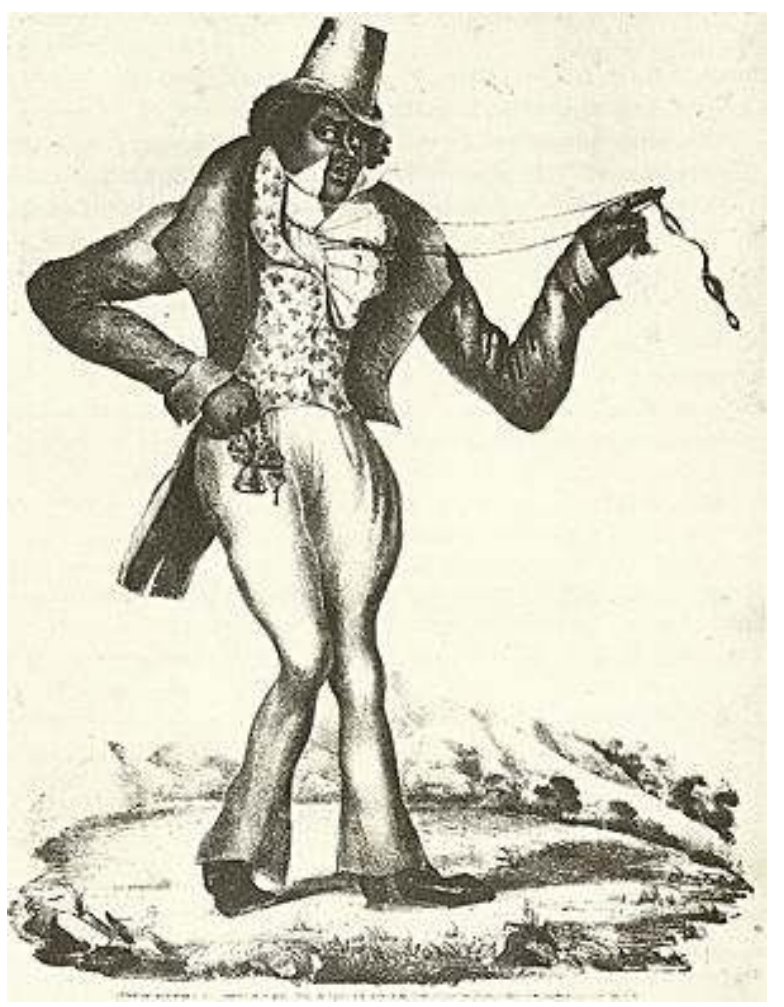

Représentation typique de Zip Coon, vers 1834 (auteur inconnu)

Malgré cette publicité raciste et le prix relativement élevé de un dollar, «Crazy Blues » se vend au-delà de toute espérance : 75000 copies en un mois dans les quartiers noirs (Stewart-Baxter, 1970, 12). Okeh Records est vite imité et dès 1922, tous les grands labels ont leur propre race series à destination du marché noir : les race records sont nés, un marché noir spécifique est constitué. Durant les deux décennies de son expansion, on dénote cinq à six millions de disques vendus annuellement à une clientèle noire dans les quincailleries, les salons de coiffure, les épiceries mais aussi en vente par correspondance (Levine, 2007, 225). On estime que ce sont environ 15000 titres qui ont été édités dans ces race series, avec une forte prédominance du blues ${ }^{14}$. Si le vocable race records n'était pas péjoratif à l'époque ${ }^{15}$, il traduit néanmoins le caractère ségrégué du marché du disque, organisé selon une ligne de couleur stricte ${ }^{16}$. Je vais ici retracer l'évolution du marché de la musique populaire américaine (et des industries culturelles qui le porte) pour montrer que ce caractère ségrégué a perduré jusqu'aux débuts du rock'n'roll, ce qui n'a bien entendu pas été sans conséquences.

Deux crises vont toucher durement l'industrie du disque d'avant le rock'n'roll, avec des répercussions d'autant plus importantes lorsque la musique était «raciale». La première de ces crises n'est autre que la crise de 1929. Affectant de manière plus dramatique encore le pouvoir d'achat des Noirs, le marché des race records s'effondre sur la période : de 1927 à 1933, le marché passe de 100 millions de dollars à 6 millions. Les maisons de disques tentent de s'adapter en baissant le prix des disques (qui passe de 75 cents environ dans les années vingt à 35 cents dans les années trente) et en ralentissant l'enregistrement et la promotion de nouveaux disques ${ }^{17}$. Les chanteuses de blues de la période étant accompagnées d'un orchestre, le coût d'une session studio était relativement élevé. Par conséquent, y compris après la crise, lorsque l'abrogation de la prohibition en 1933 et l'essor du jukebox stimulent de nouveau l'industrie du 
disque, les labels se tournent davantage vers des bluesmen qui s'accompagnent euxmêmes à la guitare, comme Robert Johnson dont l'influence sur la musique rock sera considérable. La deuxième crise qui va miner le marché des race records est la Seconde Guerre mondiale puisqu'elle entraîne à sa suite la fermeture des studios d'enregistrement pour deux longues années ${ }^{18}$. Lorsque les studios rouvrent leurs portes à la fin de la guerre, les grandes maisons de disques vont négliger leur race series et se concentrer sur des productions censées être moins « ciblées ». C'est alors que naissent un certain nombre de maisons de disques indépendantes, parmi lesquelles Chess (Chicago, Illinois), King (Cincinnati, Ohio), Vee-Jay (Gary), qui vont se spécialiser dans la musique africaine-américaine ${ }^{19}$.

Ces maisons de disques indépendantes n'emploient plus le terme de race records, tombé en désuétude pour ses connotations désormais ouvertement racistes. De nouveaux qualificatifs apparaissent, tels ebony (MGM) ou sepia (Deca, Capitol). Mais c'est bien le terme de rhythm and blues qui s'impose à la fin des années 1940, sous l'impulsion de RCA Victor. Bilboard Magazine, qui établit différents classements des ventes de disques, adopte le terme en juin 1949 sur la proposition de Jerry Wexler, alors journaliste au Bilboard. Parmi les artistes rhythm and blues, on retrouve T-Bone Walker, B. B. King, Muddy Waters, Joe Turner, Fats Domino, John Lee Hooker, Little Richard, Chuck Berry, Bo Didley... Des artistes très divers qui se caractérisent par une influence marquée du blues, du gospel et de la musique noire, la plus proche des classes populaires. Les guitares s'électrifient et les sections rythmiques sont plus appuyées. Si le rhythm and blues connaît un succès très important dans les communautés noires, grâce notamment au rôle devenu prépondérant des radios locales et des disc jokeys, le marché musical reste ségrégué. Dès lors, et bien que les maisons de disques les plus importantes aient délaissé leurs race series, elles vont chercher à tirer profit de l'engouement des adolescents blancs pour la musique noire. Elles vont le faire non pas en développant leur catalogue d'artistes noirs mais plutôt en proposant des reprises par des artistes blancs. Dès le milieu des années cinquante, la plupart des tubes rhythm and blues écrits et interprétés par des artistes noirs sont repris dans la foulée par un ou des artistes blancs qui vendent davantage. Pour ne citer que quelques exemples, «Sh-Boom », écrit et interprété par le groupe de doo-wop The Chords en 1954 est repris par le groupe canadien The Crew-Cuts la même année. Leur version restera numéro un neuf semaines. Devant un tel succès, le groupe reprend d'autres tubes des charts rhythm and blues comme " Don't Be Angry » (Nappy Brown), « Gum Drop » (The Charms) ou encore «Earth Angel» (The Penguins). De la même façon, «Shake, Rattle and Roll» de Joe Turner est repris par Bill Haley puis par Elvis Presley, qui reprendra également «Hound Dog» (Big Mama Thornton), "Tutti Frutti » ou encore "Long Tally Sally " (toutes deux des compositions de Little Richard, reprises par bien d'autres artistes qu'Elvis Presley). On pourrait objecter que ce phénomène de reprises n'était pas une nouveauté. Déjà à l'époque des spectacles de ménestrels, des comédiens blancs s'étaient appropriés le folklore noir du Sud. De plus, la tradition orale d'où est né le blues - et à sa suite le rhythm and blues - s'accommode mal de la notion de composition et donc de droits d'auteurs. Comme le note Jean-Paul Levet, le blues est « un processus continu de composition-recomposition plutôt qu'un acte individuel d'écriture, identifié et daté comme tel. Le blues singer, à chaque profération, re-crée, personnalise, s'approprie des «textes» issus de la mémoire collective» (Levet, 2010, 76). Par conséquent, le processus même de reprise/re-création serait ancré dans le code génétique de la musique noire ${ }^{20}$. Enfin, on pourrait ajouter que le blues, s'il s'agissait de la musique du 
peuple noir américain (Jones, 1997), est né d'influences très diverses allant des chants d'Afrique de l'Ouest aux ballades celtiques et anglaises jusqu'à la musique des Indiens d'Amérique. Finalement, tout cela serait à l'image de la musique populaire américaine : «l'histoire des Noirs pillant les Noirs, des Blancs pillant les Blancs, et se pillant les uns les autres » (Tosches, 2003, 39). Tout ceci est vrai, à une exception près. Dans les années cinquante, le phénomène des reprises a massivement fonctionné à sens unique, à l'exception notable de la reprise de "Crying In The Chapel » par Sonny Til and the Orioles. De plus, ces affirmations valent avant tout pour la période qui a précédé l'enregistrement de la musique et sa commercialisation - avant que la musique traditionnelle ne devienne une marchandise -, lorsque les enjeux, en termes de droits d'auteurs notamment, étaient moindres. Car si on ne saurait postuler une origine unique et essentialiste au rock (ni à aucune autre musique d'ailleurs), l'exploitation commerciale de la musique noire a lésé les artistes noirs qui, à la différence des artistes blancs, ne bénéficiaient pas d'un accès privilégié à la culture hégémonique. Ils étaient donc moins au fait de leurs droits et de ce à quoi ils pouvaient prétendre. La plupart des artistes noirs étaient ainsi payés à la session studio mais ne percevaient rien sur les éventuelles reprises de leur morceau. Pire, non seulement ils ne percevaient pas d'argent sur leurs propres compositions, mais ils subissaient la concurrence des chanteurs blancs qui reprenaient leurs chansons. Face aux moyens déployés par les maisons de disques pour promouvoir les reprises des artistes blancs et devant un marché pensé pour ces derniers, les artistes noirs étaient destinés à être les grands perdants des politiques raciales des maisons de disques.

\title{
Du blackface à Elvis Presley : produire de la musique « noire »... sans les Noirs
}

\author{
Faisons le calcul : si j'étais Noir, j'aurais vendu \\ moitié moins \\ Pas besoin de sortir du lycée pour savoir ça ${ }^{21}$. \\ Eminem, «White America », The Eminem Show, \\ Aftermath, 2002.
}

Mon hypothèse principale dans cet article consiste à affirmer que l'appropriation commerciale qui a été faite de la musique et de l'identité noires n'a pas pour autant profité aux chanteurs noirs puisque ce sont des artistes blancs qui ont intégré certains codes noirs à la subculture rock qu'ils contribuaient à créer. En cela, le rock'n'roll a entretenu une continuité avec les spectacles blackface qui entendaient présenter à un public blanc ce qu'ils prétendaient être la "culture-noire-authentique ». Si le blues n'est pas la seule influence du rock, la plupart des historiens et des critiques culturels sont d'accord pour reconnaître qu'il s'agit, avec la country, d'une des influences les plus déterminantes de cette musique. Cependant, en s'appropriant le rhythm and blues, en l'hybridant avec des influences country blanches et en nommant cette musique créolisée rock'n'roll, les industries culturelles ont entretenu une continuité du cycle blackface en préférant des Blancs qui jouent de la musique "noire " ou un dérivé - même s'ils ne jouaient bien évidemment pas que cela - à des Noirs jouant leur propre musique. Peu importe après tout que le masque et les costumes aient disparu et que les visages ne soient plus grimés puisque le but affiché est finalement similaire: produire de la blackness mais sans les Noirs. On prête d'ailleurs ces mots à Sam Phillips : 
"Si seulement je pouvais mettre la main sur un Blanc qui ait ce son et ce feeling Nègre, je me ferais des millions ». Que Sam Phillips ait réellement prononcé ces mots ou non - et il est après tout probable qu'il ne les ait jamais prononcés ${ }^{22}-$, il demeure que c'est bien la culture noire qui a été incorporée au courant dominant de la société américaine et non les Noirs qui ont été intégrés. Si Lhamon estime que l'on ne peut pas posséder les signes de la culture populaire, Reebee Garofalo et Steve Chapple semblent penser le contraire puisque leur ouvrage commun s'intitule Rock'n'Roll is Here to Pay ${ }^{23}$. Garofalo Reebee estime en effet que toute l'histoire de la musique populaire américaine depuis le vingtième siècle au moins peut être décrite en ces termes: "racines noires, fruits blancs» (Chapple et Garofalo, 1977, chap. 7): des innovations noires et une popularisation blanche (je rajouterais pour ma part un troisième terme qu'on ne retrouve cependant pas toujours: le recouvrement des origines noires). Si les innovations n'ont pas été le seul fait d'artistes noirs, il n'en demeure pas moins que l'exploitation commerciale de la musique noire a bel et bien fonctionné selon ce schéma. C'est le spectacle de ménestrels blackface qui emprunta le premier des images et des signes à la culture noire. Pour Lhamon, les spectacles de ménestrels blackface témoignent d'une "culture plébéienne" $d u x^{e}{ }^{e}$ siècle qui brouille les frontières raciales, les questions de race et de classe étant indissociables aux États-Unis ${ }^{24}$. Sans aller jusqu'à parler de melting-pot, Lhamon affirme malgré tout qu'au XIX ${ }^{e}$ siècle, une " population flottante » constituée des individus infériorisés de la société américaine ${ }^{25}$ émerge dans les quartiers mixtes du prolétariat et du lumpenproletariat qui fonctionnent comme autant de "poches incubatrices du cycle blackface " (Lhamon, 2008, 40). Parmi cette "population flottante ", on trouve ceux qu'on appelle péjorativement les white trash (littéralement « raclure blanche »). Ce terme d'argot insultant, apparu au début $\mathrm{du} \mathrm{XIX}^{\mathrm{e}}$ siècle, désigne la population blanche défavorisée du Sud que les conditions matérielles d'existence et la «dépravation morale» rendrait moins fréquentable encore (aux yeux de la société blanche bourgeoise) que les Noirs ${ }^{26}$. Aux États-Unis, ces Blancs misérables représentent une anomalie à la fois sociale et raciale. Nous pourrions dire, suivant la célèbre formule de Joan Scott à propos du genre, que la race est aux États-Unis une façon première de signifier des rapports de pouvoir. Ainsi que le note Sylvie Laurent dans Poor White Trash : La pauvreté odieuse du Blanc américain, « la locution poor white trash ne désigne pas tant un statut social qu'une catégorie morale. C'est l'étiage symbolique auquel on ne veut pas déchoir, la personnification honteuse des échecs impensables d'une population " racialement" destinée à prospérer " (Laurent, 2011, 8). Ne pouvant se reconnaître dans la blanchité hégémonique, les jeunes white trash se sont tournés vers certains traits distinctifs de la blackness pour se façonner une identité qui leur soit propre. Cela ne signifiait pas pour autant une identification aux Noirs, qui continuaient d'être brocardés dans les spectacles blackface et dans la vie de tous les jours. Il s'agissait plutôt de se construire une identité hybride, au croisement de la classe et de la race. Ni tout à fait blanche ni noire, bien qu'elle emprunte certains traits qu'elle perçoit à la blackness : "white trash». Nick Tosches affirme ainsi qu'à la place du «fantasme vieillot du Nègre d'avant guerre heureux, fiction sur laquelle se fondait le ménestrel 間...医, le fantasme parallèle d'un hillbilly ${ }^{27}$ fantasque et pittoresque émergeait simultanément pour prendre sa place dans la sous-culture du show-business du Sud » (Tosches, 2003, 186-87). Si les derniers spectacles de ménestrels blackface, avec visages noircis au charbon, disparaissent après plusieurs décennies d'agonie dans les années cinquante sous la pression de la NAACP ${ }^{28}$, on est en droit de s'interroger sur une certaine forme de survivance des pratiques blackface dans la culture hillbilly. À ce titre, 
Elvis Presley est sans doute un exemple assez paradigmatique de la continuité du cycle

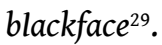

Comme la «population flottante» du blackface, Elvis Presley n'était pas tout à fait un «Blanc» au regard de la société dominante. Né en 1935 dans le Sud des États-Unis, à Tupelo, Mississippi, la famille d'Elvis Presley était relativement modeste. Mais lorsque son père est envoyé en prison en 1938 (il n'y reste que huit mois), la famille perd la maison et, dès lors, les Presley sont contraints de déménager régulièrement au gré des impératifs économiques (à chaque fois qu'ils se font mettre à la porte pour ne pas avoir payé le loyer). En 1946, la famille s'installe dans une cabane, à deux pas de Shake Rag, l'un des quartiers noirs de Tupelo. Ils sont regardés par leur voisinage comme des white trash, des hillbillies, des «bouseux ». Les camarades de classe d'Elvis ne l'entendent pas autrement, d'autant que ce dernier, qui ramène tous les jours sa guitare à l'école, ne joue et chante que de la musique hillbilly, ce qui lui vaut d'être qualifié de "raclure " (trashy) par les autres enfants blancs plus fortunés (Guralnick, 1994, 39-42). Elaine Dundy, qui a interrogé les camarades de classe d'époque d'Elvis Presley et dont les entretiens font ressortir que seuls les enfants blancs les moins fortunés semblaient apprécier Elvis, dresse un parallèle fort intéressant en affirmant que les camarades de classe d'Elvis ne l'aimaient pas davantage « qu'ils n'aimaient l'aveugle au coin de la rue chantant "Beale Street Blues" $"^{30}$ (Dundy, 2004, 123). Le statut du chanteur hillbilly (blanc) était donc similaire à celui du chanteur de blues (noir), tous deux appartenant à une race subalterne (noire et "white trash»). Vivant dans un quartier noir sans être Noir lui-même, allant à l'école avec des Blancs qui ne le perçoivent pas comme l'un des leurs, Elvis Presley personnifie le rejet qu'inspirent les white trash, décriés comme une race dégénérée, congénitalement pauvre et dont il faut se tenir éloigné :

Dans sa dimension vocative, l'apostrophe « white trash!» pourrait se traduire par «sale blanc! », ou «sale race! » lorsque le locuteur est lui-même blanc. C'est une insulte qui souligne la médiocrité sociale d'un individu dont l'appartenance raciale accentue encore la dégénérescence. (Laurent, 2011,9)

11 La prétendue « impureté raciale » d'Elvis a d'ailleurs largement été commentée ${ }^{31}$ mais c'est avec la biographie du critique Albert Goldman que l'on atteindra les sommets de cette mise en accusation raciale. Goldman questionne l'appartenance d'Elvis Presley à la race blanche et traduit la haine de classe qui s'exprime à l'encontre des white trash: « les Presley n'était pas une famille ordinaire : c'était des hillbillies... On ne peut pas imaginer une race plus déracinée et plus incapable de rester en place» (Marcus, 2003, 62). Puisque la blanchité hégémonique n'était pas un objectif atteignable pour le jeune Elvis Presley, il s'est tourné vers la musique hillbilly et la musique noire pour se façonner sa propre identité. Ainsi que nous l'avons vu, la musique hilbilly et la musique « raciale» n'étaient d'ailleurs, dans une certaine mesure, que les deux faces d'une même pièce «sale » aux yeux de la société blanche dominante ${ }^{32}$. En devenant "white trash", Elvis Presley s'est également "fait Noir » jusqu'à un certain point. Et c'est justement parce qu'il connaissait extrêmement bien la culture noire avec laquelle il entretenait un lien privilégié qu'il a pu s'identifier à elle. En effet, au gré de ses multiples déménagements, la famille Presley a toujours vécu soit à l'entrée des quartiers dits de couleur soit directement dans ces quartiers. Elvis Presley pouvait donc observer la culture noire du pas de sa porte. Selon des témoignages de première main recueillis par Greil Marcus, Elvis Presley se rendait d'ailleurs à Memphis dans des clubs dans lesquels se produisent des artistes noirs. Un promoteur de Beale Street affirme 
ainsi avoir emmené Elvis Presley dans l'un de ces clubs et observé comment Elvis a développé ses propres performances de la blackness :

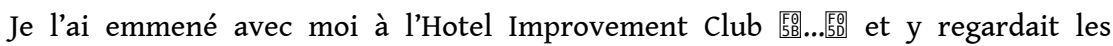
chanteurs de couleur, comprenez-moi bien, et y se mettait à faire comme eux. Il a pris cette façon de se trémousser, ce déhanchement de Charlie Burse, Ukelele Ike qu'on l'appelait, juste là au Gray Mule, sur Beale. Elvis, y faisait rien de plus que ce que les gens de couleur faisaient depuis cent ans. (Marcus, 2003, 71)

Y compris dans sa façon de parler, Elvis Presley avait absorbé des influences «noires » ou, en tout cas, ce qu'il percevait de la blackness. Outre le bégaiement en début de phrase (Tosches, 2003, 134), on retrouve chez Elvis Presley un certain "parler noir", comme par exemple dans l'interlude parlée (une des caractéristiques des chansons blackface que l'on retrouve parfois dans la musique country) de "I Love You Because ». Elvis Presley, qui s'est avant tout fait connaître par ses reprises de classiques blues, rhythm and blues et country, avait infusé sa connaissance de la culture noire dans ses enregistrements tout en laissant transparaître sa propre blanchité " white trash ». C'est d'ailleurs pour cette raison qu'Elvis Presley apparaissait subversif aux yeux des ÉtatsUnis ségrégationnistes : «le rock'n'roll devint la cible des ségrégationnistes du Sud qui

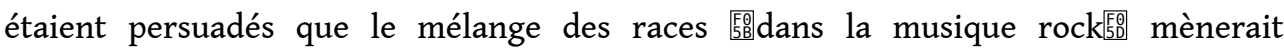
inévitablement au métissage et que l'exposition à la culture noire favorisait la délinquance juvénile et allait contre la morale sexuelle $»^{33}$ (Altschuler, 2003, 37). Elvis Presley, traître à la race blanche, était donc une cible privilégiée, d'autant plus qu'il reconnaissait sa dette à l'égard des artistes Noirs, comme dans cette interview pour le Charlotte Observer en 1956 :

Les gens de couleur chantaient et jouaient [le rock'n'roll] exactement comme je le fais aujourd'hui, et depuis plus longtemps que je ne saurais dire. Ils jouaient cette musique dans les shanties et dans les juke joints. Et personne n'y faisait gaffe jusqu'à ce que j'en fasse de même. C'est d'eux que je le tiens ${ }^{34}$. (cité dans Altschuler, 2003, 30)

13 Elvis est ici lucide sur les politiques raciales de l'industrie du disque américaine, reconnaissant que ce n'est que lorsque la musique noire est apparue moins «noire » qu'elle a pu connaître le succès qu'on lui connaît. Elvis Presley a donc contribué à intégrer des codes noirs à la subculture rock puis, par la suite, à l'ensemble de la société américaine. Mais encore une fois, ce sont bien les signes noirs qui ont été intégrés plus que les Noirs eux-mêmes ${ }^{35}$, même si certains ont pu bénéficier marginalement de l'engouement nouveau pour le blues de la part des Américains blancs.

\title{
Masques blackface et injonction à l'authenticité raciale : la « redécouverte » d'artistes africains- américains dans les années soixante
}

\author{
"On arrête de jouer du blues. Laissons le blues \\ aux Blancs. Ils s'en sont emparés, eh bien qu'ils le \\ gardent. Joue autre chose $»^{36}$ \\ Miles Davis à Herbie Hancock (Maher Jr. et Dorr,
} 2009, 41).

Dans un ouvrage majeur des cultural studies, Dick Hebdige explique que l'ordre est constamment menacé par l'altérité que représentent les subcultures. La société 
dominante a alors deux options, deux stratégies, qui s'offrent à elle: «d'une part, l'Autre peut être banalisé, naturalisé et domestiqué. Dans ce cas, la différence est tout simplement niée, l'altérité est réduite à l'identité. Inversement, l'Autre peut être transformé en phénomène exotique au-delà du sens" (Hebdige, 2008, 102). Il me semble qu'avec Elvis Presley et plus largement le rock'n'roll blanc, nous sommes en face de la première des stratégies décrites par Hebdige : l'incorporation au courant dominant de la société américaine a permis, sinon de domestiquer totalement la subculture rock ${ }^{37}$, de lui enlever sa connotation raciale. Du rock'n'roll oui, mais pas du rhythm and blues. Des influences noires mais des artistes blancs. L'ordre subverti est rétabli. Comme le notait déjà Barthes en 1957, l'Ordre ne peut reconnaître l'altérité qu'à condition de la désamorcer en la transformant en «pur reflet » de lui-même (Barthes, 1970, 154). Quant aux artistes noirs qui ont si fortement influencé et même façonné le rock'n'roll des débuts, ils seront rejetés hors du sens et du temps, transformés en " pur objet, spectacle, guignol» (Barthes, 1970, 226). Ainsi, lorsque ces artistes vont être "redécouverts " par un public blanc dans les années soixante (par le biais de la scène folk aux États-Unis ou du British Blues Boom en Angleterre), il leur sera demandé de performer une blackness fantasmée, sans lieu ni histoire véritable mais considérée comme "authentique». Si les Blancs américains ne s'intéressent que tardivement au blues, cette musique est de plus en plus plébiscitée au début des années soixante, grâce notamment au mouvement folk urbain (dont Dave Van Ronk et surtout Bob Dylan comptent parmi les plus éminents représentants). Cet intérêt tardif témoigne d'une nouvelle attitude de la jeunesse américaine qui rejette tout à la fois les crooners, les chansons de Tin Pan Alley ${ }^{38}$ et le rock'n'roll devenu trop commercial à son goût. Ce que cette jeunesse recherche, c'est l'authenticité : la guitare acoustique revient à la mode et l'on découvre et redécouvre tout un pan du folklore américain, celui des États du Sud et des Noirs notamment. Dans les campus, le blues bénéficie d'un énorme engouement. Cependant, si le blues revival va contribuer à sortir des artistes noirs de l'anonymat dans lequel ils étaient retournés, le goût pour une supposée authenticité va figer le blues hors du temps. Alors que cette musique avait évolué et donné naissance au rhythm and blues, les Blancs libéraux américains se désintéressaient largement du blues électrique comme pratiqué à Chicago par exemple, celui-là même auquel le rock'n'roll devait tant, préférant déterrer les enregistrements de country blues réalisés par des folkloristes qui correspondaient davantage "à leur quête des notions de "vérité", de "vitalité" et d' "authenticité ethnique” " (O'Neal, 1994, 378). Le country-blues est seul jugé " authentique » et ce sont par conséquent les artistes qui se plient au goût du public blanc qui bénéficient de ce mouvement de redécouverte. L'injonction à l'authenticité amène un certain nombre d'artistes noirs comme John Lee Hooker ou Lightnin' Hopkins à abandonner guitares électriques et orchestres pour retourner à une forme de blues plus primitive. Les chanteurs de blues, s'ils souhaitent « réussir » commercialement, doivent désormais se soucier de plaire non pas à leur public noir traditionnel mais à un public de nouveaux amateurs blancs. Alors que le blues était depuis les origines associé à l'expérience des Noirs aux États-Unis, qu'il était une "propriété collective " pour reprendre l'expression d'Angela Davis dans Blues Legacies and Black Feminism, les années soixante voient arriver un nouveau public qui, s'il est certes bienveillant, reproduit malgré lui des stéréotypes racisés, attendant des artistes noirs qu'ils donnent à voir un passé fantasmé. Car cette quête d'authenticité des Blancs libéraux suppose pour les artistes noirs de performer une blackness primitive, qui n'est pas sans rappeler « une 
émanation du plus grossier et du plus dégradant des avatars du ménestrel », ainsi que le note Nick Tosches :

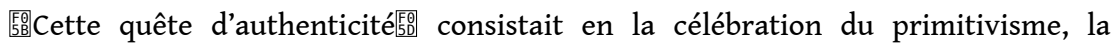
romance de la rusticitas par des Américains jeunes, « libéraux » et blancs, cherchant à échapper à la vacuité à travers la trompeuse pseudo-négritude de la « vérité crue et dure » du blues. (Tosches, 2003, 119)

Déjà à l'époque du blackface, les comédiens noirs qui se produisaient dans les spectacles de ménestrels se grimaient eux aussi le visage au charbon pour servir à leur audience le frisson racial attendu. Cela atteste du fait qu'un Noir n'était jamais suffisamment «noir», «authentiquement» noir, aux yeux du public blanc ${ }^{39}$. Concernant le blues, l'injonction à l'authenticité raciale produisait les mêmes attentes. Ce qu'on attendait des chanteurs de blues "redécouverts", c'était qu'ils performent leur rôle de Noir sudiste analphabète travaillant au champ de coton. De manière très révélatrice, le chanteur de blues redécouvert était d'ailleurs quasiment toujours de sexe masculin, comme si la blackness " authentique " ne pouvait se conjuguer au féminin. Lawrence Cohn, qui a dirigé un ouvrage de référence sur le blues, note à ce propos : « Vous savez, c'était le mythe du vieux paysan, le Noir peinant sous le soleil de midi, gémissant et

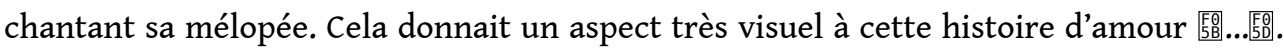
Et puis, il y avait le frisson de la redécouverte de ces gens proprement incroyables » (O'neal, 1994, 378). Frantz Fanon, dans les pages consacrées à la "culture nationale " des Damnés de la terre, note que c'est le colonisateur qui est le plus grand défenseur du style et des traditions indigènes mais qu'il les préfèrent figées hors de toute histoire. Pour appuyer son propos, il revient sur les réactions de certains «spécialistes » blancs du jazz à l'égard du be-bop et déconstruit par là les ressorts de l'injonction à l'authenticité raciale :

On se souvient parfaitement, et l'exemple revêt une certaine importance parce qu'il ne s'agit pas tout à fait d'une réalité coloniale, des réactions des spécialistes blancs du jazz quand, après la Deuxième Guerre mondiale, se cristallisèrent de façon stable de nouveaux styles comme le be-bop. C'est que le jazz ne doit être que la nostalgie cassée et désespérée d'un vieux nègre pris entre cinq whiskies, sa propre malédiction et la haine raciste des Blancs. Dès lors que le nègre s'appréhende et appréhende le monde différemment, fait naître l'espoir et impose un recul à l'univers raciste, il est clair que sa trompette tend à se déboucher et sa voix à se désenrouer. (Fanon, 1961, 181)

16 Aux États-Unis, les implications racistes de l'injonction à l'authenticité raciale n'échappaient bien entendu pas aux yeux des principaux concernés. Si certains artistes noirs ont porté le masque blackface pour satisfaire les goûts de leur nouveau public blanc et s'offrir une nouvelle carrière, ils n'étaient pas dupes. D'après un compte-rendu du magazine Rhythm and Blues de 1963, Lightnin' Hopkins avait conscience que son public le considérait avant tout comme un phénomène de foire, "exotique ", et c'est pourquoi il n'offrait qu'une partie de lui-même. Memphis slim expliquait quant à lui que le public blanc était un public bien plus facile à conquérir que le public noir qui, lui, avait une réelle connaissance du blues (Levine, 2007, 233). Le succès tenait donc à la capacité d'un artiste noir à répondre aux injonctions à la blackness du public blanc. C'est pourquoi Muddy Waters affirmait qu'il préférait jouer en Europe car là bas, on ne lui demandait pas de faire l'Oncle Tom $^{40}$ ni le bouffon. Et justement, le personnage de l'Oncle Tom a été une influence première dans beaucoup de rôles des spectacles ménestrels blackface. On voit ainsi se tisser les liens entre injonction à l'authenticité raciale et masque blackface dans le caractère avilissant que tous deux revêtent ${ }^{41}$. 
Le commentaire de Muddy Waters m'amène pour finir à m'intéresser à l'importation du blues en Angleterre, en me demandant si les logiques à l'œuvre ont été les mêmes làbas. Dès 1962, l'Europe découvre le blues grâce à l'American Folk Blues Festival ${ }^{42}$ et par le biais d'artistes tels que John Lee Hooker, Memphis Slim, Willie Dixon ou encore TBone Walker. Toute une génération de Britanniques se passionne pour le blues, acoustique et électrique. Si la jeunesse connaissait déjà le rock'n'roll, elle ignorait cependant quasiment tout de ses origines noires. Dès lors et contrairement à ce qui s'est passé aux États-Unis, où la musique a toujours été très fortement ségréguée, les jeunes groupes britanniques qui se montent et reprennent des standards noirs américains vont se montrer plus soucieux de ne pas renier les racines noires. Ainsi, les Rolling Stones nomment leur groupe d'après une chanson de Muddy Waters et affirment que la musique qu'ils jouent est du rhythm and blues et non du rock, comme pour se démarquer du rock'n'roll blanc américain. Si les Stones empruntent très largement des chansons et des signes à la culture noire, ils ne taisent cependant pas leurs influences, citant les noms de chanteurs de blues à longueur d'interviews et réclamant par exemple la présence d'Howlin' Howlf pour accepter de passer à l'émission «Shindig » en $1965^{43}$. La subculture blues britannique devient un mode de vie et une façon d'appréhender le monde. Keith Richards se montre lucide sur l'ambivalence d'une telle identification: «Quand on y réfléchit, un imbécile blanc boutonneux de dix sept ans de Dartford qui veut devenir Muddy Waters - et on était beaucoup à le vouloir - ça a quelque chose de pathétique dans un sens. Mais d'un autre côté... ça fait chaud au cœur $»^{44}$. Si le « prestige noir » décrit par Lhamon a fonctionné à plein régime en Grande-Bretagne, il est important de rappeler que malgré le respect témoigné par les artistes blancs aux chanteurs de blues noirs, seule une minorité de ces derniers bénéficièrent effectivement du blues revival. Ce sont essentiellement les artistes blancs, et notamment les Britanniques, qui ont bénéficié de cet engouement de la jeunesse pour le blues, les artistes noirs restant le plus souvent à la marge ou triés sur le volet, comme gage d'authenticité. Ainsi, chaque maison de disque voulait son propre groupe de blues électrique, mais un groupe composé de Blancs chantant le répertoire noir du blues. Si le blues, musique noire, a été reconnu en Angleterre comme l'influence première du rock'n'roll (là-bas, la country n'était que peu connue et encore moins appréciée), ce sont malgré tout les jeunes groupes blancs qui ont raflé la mise en performant un idiome musical largement noir. Racines noires, donc, mais fruits blancs.

\section{Conclusion}

18 Je n'ai pas cherché dans cet article à opposer une musique noire authentique à une musique blanche qui ne serait que pillage car il me semble qu'on retombera toujours dans l'essentialisme en prenant l'authenticité comme critère d'évaluation ${ }^{45}$. En explorant une généalogie de la musique rock, de ses origines et de ses circulations transatlantiques, j'ai simplement essayé de mettre en évidence un certain nombre de politiques raciales de la musique populaire américaine $d u x^{e}$ siècle. Mon propos principal était de montrer les imbrications fortes qui existent entre le blackface et l'exploitation commerciale de la blackness dans le rock'n'roll des débuts, ce qui me permet de postuler une continuité du cycle blackface dans la musique populaire américaine d'après la seconde guerre mondiale. Si la culture noire a été incorporée au courant dominant de la société américaine, on l'a pourtant largement « dé-racialisée ». 
L'appropriation de la blackness par des Blancs remonte certes aux spectacles de ménestrels en blackface, mais l'abandon du visage noirci au charbon dans les années cinquante a troublé les processus d'identification raciaux. À l'époque des spectacles de ménestrels blackface, les comédiens blancs qui se noircissaient le visage ne devenaient «Noirs» qu'une fois grimés. Il ne faisait donc aucun doute que la blackness était «noire ». Avec la subculture rock, les performances de blackness ne sont plus mises à distance : un Blanc peut se faire « Noir " sans avoir à se noircir le visage. La blackness n'est plus «chromatisée ", elle peut même désormais être "blanche ». C'est pourquoi j'affirme que c'est la culture noire qui a été intégrée plus que les Noirs eux-mêmes, bien que certains d'entre eux aient pu bénéficier d'un soudain intérêt pour l'« exotisme » qu'ils représentaient. Cela à condition de se conformer aux goûts de leur nouveau public blanc. De la performance d'une blackness sans les Noirs (peau blanche, masques noirs) à l'injonction à l'authenticité raciale pour les artistes noirs " redécouverts » (peau noire et masques «noirs »), l'histoire du rock est indissociable de ses pratiques blackface.

\section{BIBLIOGRAPHIE}

ALTSCHULER, Glenn C., All Shook Up - How Rock'n'Roll Changed America, New York, Oxford University Press, 2003.

BARLOW, William, « Cashing in: 1900-1939 », dans William Barlow et Janette Dates, dirs., Split image - African Americans in the Mass Media, Washington, Howard University Press, 1993.

BARTHES, Roland, Mythologies, Paris, Éditions du Seuil, 1970.

BEAN, Anne-Marie et al., dir., Inside The Minstrel Mask - Readings in Nineteenth-Century Blackface Minstrelsy, Hanover, Wesleyan University Press, 1996.

BENJAMIN, Walter, Euvres, III, Paris, Gallimard, 2000.

BHABHA, Homi, Les lieux de la culture - Une théorie postcoloniale, Paris, Payot, 2007.

BRADFORD, Perry, Born with the Blues - Perry Bradford's Own Story, New York, Oak Publications, 1965.

CHAPPLE, Steve et Reebee GAROFALO, Rock'n'Roll Is Here to Pay - The History and Politics of the Music Industry, Chicago, Nelson-Hall, 1977.

DUNDY Elaine, Elvis and Gladys, Jackson, University Press of Mississippi, 2004.

FANON, Frantz, Les damnés de la terre, Paris, Éditions Maspero, 1961.

FLEMING, Thomas, «Black Musicians and Early Radio », The Red Hot Jazz Archive, http:// www.redhotjazz.com/smitharticle.html (page consultée le 15 octobre 2013).

GURALNICK, Peter, Elvis Presley. Last Train To Memphis - Le temps de l'innocence (1935-1958), Paris, Le Castor Astral, 1994. 
HALL, Stuart et Tony JEFFERSON, dirs., Resistance through Rituals - Youth Subcultures in Post-war Britain, Londres, Routledge, 2004.

HALL, Stuart, Identités et cultures - Politiques des cultural studies, Paris, Éditions Amsterdam, 2008.

---, Identités et cultures 2 - Politiques des différences, Paris, Éditions Amsterdam, 2013.

HEBDIGE, Dick, Sous-culture - Le sens du style, Paris, Zones, 2008.

JONES, LeRoi, Le peuple du blues, Paris, Gallimard, 1997.

KEIL, Charles, Urban Blues, Chicago, University of Chicago Press, 1966.

KILLMEIER, Mathew, « Race Music », St. James Encyclopedia of Pop Culture, vol. 4, 2002.

LAURENT, Sylvie, Poor White Trash - La Pauvreté Odieuse du Blanc Américain, Paris, Presses de

l'Université Paris-Sorbonne, 2011.

LEVET Jean-Paul, Talkin' that talk - Le langage du blues, du jazz et du rap, Paris, Éditions Outre Mesure, 2010.

LEVINE, Lawrence, Black culture and Black Consciousness - Afro-American Folk Thought from Slavery to Freedom, New York, Oxford University Press, 2007.

LHAMON, William, Peaux blanches, masques noirs - Performances du Blackface de Jim Crow à Michael Jackson, Paris, Kargo \& l'Éclat, 2008.

LOTT, Eric, Love \& Theft - Blackface Minstrelsy and the American Working Class, New York, Oxford University Press, 1993.

MAHER Jr., Paul et Michael DORR, Miles on Miles. Interviews and Encounters with Miles Davis, Chicago, Lawrence Hill Books, 2009.

MARCUS, Greil, Mystery Train - Images de l'Amérique à travers le rock'n'roll, Paris, Éditions Allia, 2001.

---, Dead Elvis - Chronique d'une obsession culturelle, Paris, Éditions Allia, 2003.

MILLER, Karl Hagstrom, « Race Records And Old-Time Music. The Creation of Two Categories in the 1920s ", dans Segregating Sound - Inventing Folk and Pop Music in the Age of Jim Crow, Durham, Duke University Press, 2010.

NEWITZ, Annalee et Matt WRAY, White Trash - Race and Class in America, New York, Routledge, 1997.

O'NEAL, Jim, « Standing at the crossroads : les blues aujourd'hui », dans Lawrence Cohn, dir., Nothing but the Blues. Le Blues, sa musique et ses musiciens, Paris, Abbeville, 1994.

SIMOND, Ike, Old Slack's Reminiscence and Pocket History of the Colored Profession from 1865 to 1891, Bowling Green, Popular Press, 1974.

SOUTHERN, Eileen, Histoire de la musique noire américaine, Paris, Buchet/Chastel, 1976.

STEWART-BAXTER, Derrick, Ma Rainey and the Classic Blues Singers, New York, Stein\&Day, 1970. TOLL, Robert, Blacking Up - The Minstrel Show in Nineteenth Century America, New York, Oxford University Press, 1974.

TOSCHES, Nick, Country - Les raciness tordues du rock'n'roll, Paris, Éditions Allia, 2000.

---, Blackface - Au confluent des voix mortes, Paris, Éditions Allia, 2003.

VAN RONK, Dave et Elijah WALD, Manhattan Folk Story, Paris, Robert Laffont, 2013. 


\section{NOTES}

1. La traduction est de Sophie Renaut (Lhamon, 2008).

2. J'emprunte cette expression à Robert Toll qui titre ainsi son étude sur les spectacles de ménestrels blackface dans les États-Unis du XIX ${ }^{e}$ siècle (Toll, 1977). Plus récemment, le groupe de rap africain-américain Shabazz Palaces titrait son premier album Black Up. Alors que chez Robert Toll, "blacking up" signifie "se faire Noir», le titre de Shabazz Palaces a des implications différentes. Il s'agit d'un discours performatif prônant la fierté noire, dans la lignée de James Brown et de son « Say It Loud - I'm Black and Proud ». Dans cet article, «blacking up » signifiera une mise en scène consciente d'une certaine idée de la blackness qui est performée devant un public majoritairement blanc.

3. Il convient ici de préciser que le blackface n'est pas une forme théâtrale en soi mais une pratique. Indissociable du spectacle de ménestrels ; on ne saurait néanmoins réduire le blackface à cette seule forme théâtrale puisqu'on en retrouve des avatars dans le vaudeville, la comédie musicale, le cinéma... C'est d'ailleurs mon ambition dans cet article que de montrer que la pratique blackface - avec ou sans maquillage - a survécu aux spectacles de ménestrels. Pour une discussion passionnante des liens entre les spectacles de ménestrels et la pratique du blackface, lire Eric Lott, Love \& Theft - Blackface Minstrelsy and the American Working Class, New York, Oxford University Press, 1993.

4. «My project is to examine that instability for what it may tell us about the racial politics of culture in the years before the Civil War ». Il s'agit de mes traductions, sauf mention contraire. Chaque traduction est accompagnée en note de bas de page de la version originale.

5. Je développe cet aspect dans la deuxième partie de cet article, à propos de la locution white trash et des identifications raciales troublées qui en résultent.

6. Par blackness, j'entends tous les prétendus traits caractéristiques que l'on prête, indistinctement, aux Noirs et, plus que cela, au fait d' « être Noir ». La blackness est ce qui définit l'identité noire et en un sens, elle est l'identité noire. Ainsi que le note Stuart Hall, l'identité noire est « un récit, un conte, une histoire ; quelque chose de construit, d'énoncé, de raconté, et non quelque chose que l'on trouve » (Hall, 2013, 21). C'est pourquoi, en tant que système complexe de signes, la blackness ne connaît pas une définition unique mais est un enjeu de luttes en termes de représentations hégémoniques.

7. De plus, et ainsi que le note Lawrence Levine, après l'esclavage et plus particulièrement au tournant $\mathrm{du} \mathrm{XIX}^{\mathrm{e}}$ siècle et $\mathrm{du} \mathrm{Xx}^{\mathrm{e}}$ siècle, les frontières entre musiques "noire » et «blanche » se sont faites plus poreuses. Les spectacles de ménestrels blackface ont joué un rôle central à cet égard puisque, bien avant le rock, les comédiens blancs blackface arpentaient le sud pour apprendre des chansons du folklore noir qu'ils reprenaient ensuite dans leur spectacle et popularisaient dans le reste du pays. En retour, des Noirs découvraient certaines chansons du folklore noir du Sud par le biais de ces comédiens blancs (Levine, 2007, 192).

8. J'utilise l'expression subculture plutôt que la traduction "sous-culture » que l'on retrouve parfois en raison de la confusion qu'une telle traduction entretient, en français, entre subculture [sous-culture] et low culture [basse culture]. Par subculture, il faut entendre une culture significativement différente (en termes de valeurs, d'activités, de lieux fréquentés, etc.) de la société prise dans son ensemble. Les subcultures sont toujours ramenées à un groupe qui en est le garant. Lorsque ce groupe est défini par l'âge ou par une génération, on parle alors de subcultures jeunes ou juvéniles. Pour des éléments de définition plus poussés, je renvoie à Stuart Hall et Tony Jefferson (2004, 13-17).

9. «There's fourteen million Negroes in our great country and they will buy records if recorded by one of their own ».

10. En réalité, «Crazy Blues » est issu de la deuxième séance studio de Mamie Smith. En janvier 1920, la chanteuse avait déjà enregistré pour le même label « That Thing Called Love » ainsi que 
«You Can't Keep a Good Man Down ». Le disque, s'il n'avait pas été promu par Okeh Records, avait rencontré un succès suffisamment important pour que le label renouvelle l'expérience.

11. Pour une analyse plus détaillée des publicités de race records au prisme du blackface, voir MILLER, Karl Hagstrom, « Race Records And Old-Time Music. The Creation of Two Categories in the 1920s ", in Segregating Sound - Inventing Folk and Pop Music in the Age of Jim Crow, Durham, Duke University Press, 2010.

12. Zip Coon est un personnage récurent des spectacles de ménestrels blackface. Fainéant, maladroit, efféminé (dominé par sa femme, selon le mythe raciste et sexiste du matriarcat noir) et parlant un anglais approximatif, il est censé incarner le dandy noir urbain. On retrouve des avatars de Zip Coon dans nombre de publicités de race records.

13. «I's telling you Mamie's beats 'em all ».

14. Environ 10000 disques de blues, 3250 de jazz et 1750 de gospel (Killmeier, 2002).

15. «Le mot "race" symbolisait la fierté noire, le militantisme et la solidarité dans les années vingt, et il était généralement préféré à "de couleur" (colored) ou "Nègre" (Negro) par les Africains-Américains urbains » (Barlow, 1993, 38). « Race was symbolic of black pride, militancy, and solidarity in the 1920s, and it was generally favored over colored or Negro by AfricanAmerican city dwellers ».

16. Tout comme les États-Unis sont partagés entre le Nord et le Sud par la «ligne de couleur » (Color Line) le marché musical est lui aussi organisé selon une ligne semblable (Miller, 2010).

17. Les maisons de disques adoptent alors une stratégie fréquemment reprise depuis: la réédition et la publication d'inédits, dont les coûts sont bien moindres.

18. Le shellac, indispensable à la production des 78 tours, est produit en quantité extrêmement limitée durant la guerre. De plus, une grève illimitée est déclarée en 1942 par le syndicat de l'American Federation of Music qui estime que les jukebox mettent en difficulté les orchestres live.

19. Il est intéressant de noter que King Records a d'abord été spécialisé dans la musique country, comme si le passage de la country à la musique noire présentait une certaine cohérence, ce qui pourrait s'expliquer par les ressemblances entre ces deux musiques, la country ayant été influencée par la musique noire et l'ayant influencée en retour (Levine, 2007, 274). Nick Tosches estime que ce sont les spectacles de ménestrels qui ont constitué «l'inspiration et le terreau

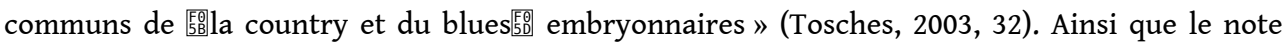
Miller dans Segregating Sound (2010), la musique du Sud n'était pas, avant les années vingt, définie racialement, avec les Noirs jouant le blues et les Blancs sudistes jouant la country. Ce sont les maisons de disques qui apposèrent de telles étiquettes pour nourrir leurs catalogues en race et hillbilly records. Il convient ici d'observer que la musique country était elle aussi ségréguée, coupée du reste de la production discographique blanche.

20. Il serait plus juste de dire qu'un tel processus est à la base de toutes les musiques traditionnelles, ce qui inspira ce bon mot au folkloriste Paul Clayton: «Si tu sais pas écrire, réécris. Si tu sais pas, dépose le copyright » (Van Ronk et Wald, 2013, 150). « If you can't write, rewrite. If you can't rewrite, copyright ».

21. «Let's do the math: if I was Black, I would've sold half / I ain't have to graduate from Lincoln high school to know that ".

22. Sam Phillips a toujours nié avoir jamais prononcé cette phrase, arguant que son ambition était de réunir les deux races et leur musique. Par ailleurs, la secrétaire de Sam Phillips qui aurait rapporté ces propos, Marion Keiser, affirme n'avoir jamais entendu son ancien patron employer le mot « nègre » : « rien ne lui ressemble moins 臤...医. Jamais. Jamais - jamais. Je ne crois pas que Sam ait jamais utilisé ce mot de sa vie et il ne l'a certainement jamais utilisé en ma présence » (Marcus, 2003, 67).

23. Jeu de mots sur la chanson « Rock'n'roll is here to stay » de Danny and the Juniors.

24. Il ne faut pas entendre derrière "brouiller les frontières raciales» la disparition de ces frontières, mais plutôt une porosité plus marquée. Le spectacle de ménestrels blackface a troublé 
les identifications raciales, les rendant plus ambigües et moins automatiques : " prendre la place

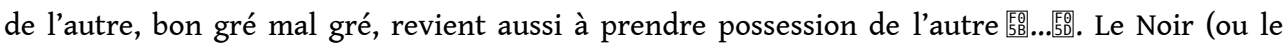
Blanc) s'immisce dans l'autre, comme un caillou dans la chaussure » (Lhamon, 2008, 48-49); le

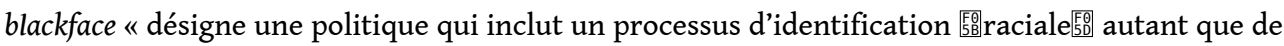
différenciation » (Ibid., 341-42).

25. "Le Chatham renfermait un espace scénique rudimentaire que se partageaient, à Catherine Slip, les maraîchers, les esclaves, les Noirs affranchis, les bouchers, les marins, les ciseleurs, les terrassiers et autres journaliers de toutes les couleurs. Tous faisaient l'expérience d'une difficile liberté » (Lhamon, 2008, 65).

26. Pour une généalogie du terme et une analyse de ses implications raciales, voir Annalee Newitz et Matt Wray, White Trash : Race and Class in America, New York, Routledge, 1997.

27. Terme d'argot, péjoratif, servant à l'origine à désigner les habitants des Appalaches, réputés idiots et aux mœurs consanguines. Une traduction possible est "péquenaud ». Hillbilly donnera son nom à la musique de cette région, avant d'être supplanté par l'expression country and western.

28. La NAACP désigne la « National Association for the Advancement of Colored People ». Fondée en 1909, elle est la plus ancienne et la plus influente des associations de défense des droits des Africains-Américains.

29. Plus tard, Eminem jouera un rôle assez similaire à celui d'Elvis Presley, comme il le reconnaît ironiquement dans « Without Me » : "Je suis la pire chose qui soit arrivée depuis Elvis Presley / À faire de la musique noire de manière si égoïste / et m'en servir pour devenir riche / Hey, c'est un concept qui marche! » («I am the worst thing since Elvis Presley / To do black music so selfishly / And use it to get myself wealthy / Hey, there's a concept that works »). Eminem, "Without Me ", The Eminem Show, Aftermath, 2002. La pochette représente d'ailleurs Eminem sur une scène de théâtre, derrière des rideaux rouges. Couplée au nom de l'album - The Eminem Show -, il est difficile de ne pas faire le rapprochement avec les spectacles de ménestrels.

30. «His schoolmates would no more love Elvis than they would love the blind man on the corner singing "Beale Street Blues" ».

31. Avec un sens du détail génétique et biologique qui confine pour le moins au culturalisme le plus absolu et même au racisme, comme dans la biographie d'Elaine Dundy: «Du point de vue génétique, Elvis était le résultat d'un sacré mélange. Pour commencer, du sang français normand ajouté à du sang écosso-irlandais. Et quand vous ajoutez à ça une ascendance indienne qui apporte du mystère et des origines juives pour le sens du spectacle, que vous liez tout ça avec son environnement, son milieu social et son éducation religieuse - surtout cette religion des pauvres blancs du Sud, la Première Assemblée de Dieu - vous avez le mystère qu'était Elvis » (Dundy, 2004, 26). "Genetically speaking, what produced Elvis is quite a mixture. At the beginning, to French Norman blood was added Scots-Irish blood. And when you then add to these the Indian strain supplying the mystery and the Jewish strain supplying spectacular showmanship, and you overlay all this with his circumstances, social conditioning, and religious upbringing-specifically his Southern poor white, First Assembly of God upbringing-you have the enigma that was Elvis ».

32. Lawrence Levine rappelle que la musique country a toujours occupé une place singulière dans le paysage musical blanc américain : «Les alternatives existaient non seulement pour les groupes ethniques mais aussi pour ceux dont les goûts et les valeurs étaient représentés dans la musique country-western. Comme la musique noire qui l'a profondément influencée, la musique countrywestern traitait de manière beaucoup plus explicite de sujets tels que le sexe, le divorce, l'alcool, et de ce que l'un de ses morceaux appelait Les Dures réalités de la vie (The Cold Hard Facts of Life), que ne le faisait la musique populaire américaine » (Levine, 2007, 274). « Alternatives existed not only for ethnic groups but for those whose tastes and values were represented in country-western music which, like the black music that profoundly influenced it, also dealt much more explicitly 
with such subjects as sex, divorce, drink, and what one of its songs called The Cold Hard Facts of Life than did American popular music ».

33. « Rock ' $n$ ' roll became a target of southern segregationists, who believed that race mixing led, inevitably, to miscegenation and that exposure to black culture promoted juvenile delinquency and sexual immorality ».

34. " The colored folks been singing it and playing it [rock'n'roll] just like I'm doing now, man, for more years than I know [...]. They played it like that in the shanties and in their juke joints and nobody paid it no mind until I goose it up. I got it from them $»$.

35. Il convient d'ailleurs de signaler que malgré ces déclarations et l'admiration qu'Elvis Presley portait à Arthur «Big Boy » Crudup, l'auteur de «That's All Right » - qui deviendra " That's All Right, Mama » lorsque reprise par Presley - ne toucha jamais aucun droit d'auteur sur ces chansons et mourut dans la misère en 1974.

36. "We're not going to play the blues anymore. Let the white folks have the blues. They got 'em, so they can keep 'em. Play something else ».

37. En tant que subculture fédératrice de la jeunesse blanche des années cinquante, le rock des premières années présentait un caractère potentiellement subversif, à cause de ses implications raciales et sexuelles, même si fortement ancré dans la culture consumériste. Les débuts d'Elvis Presley au cinéma signent un premier point culminant du processus d'incorporation de la subculture rock au courant dominant.

38. Du nom du centre de New York où s'étaient regroupés les éditeurs musicaux à la fin du XIX siècle. Par extension, « Tin Pan Alley » est devenu le nom donné à la musique de variété.

39. Voir l'interprétation tout à fait intéressante que livre LeRoi Jones dans Le Peuple du blues. Selon Jones, la caricature raciste livrée par les spectacles blackface en dit davantage sur la société blanche que sur les Noirs : «l'imitation ou la caricature de ce qui parait typique chez l'homme

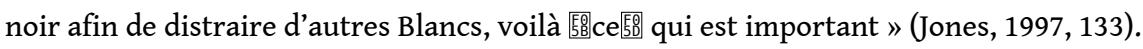

40. L'épithète Oncle Tom, forgé d'après le personnage du roman de Harriett Beecher Stowe, désigne un Noir servile et dévoué aux Blancs dont le comportement est jugé avilissant. On dit alors "agir comme un Oncle Tom». Cette expression est passée dans le vocabulaire courant américain et constitue une injure particulièrement forte dans la communauté noire.

41. Sam Lucas, l'un des plus célèbres comédiens noirs blackface de la seconde moitié du XIXe siècle, célèbre pour ses interprétations de l'Oncle Tom, confia un jour à son collègue Old slack que plus jamais il ne souhaitait se noircir le visage, ce qui atteste de la conscience des artistes noirs du caractère avilissant qu'il y a à se grimer (Simond, 1974, 9). En 1914, âgé de 72 ans et retiré des spectacles de ménestrels blackface depuis plusieurs années, il reprit le rôle de l'Oncle Tom pour l'adaptation cinématographique du roman de Harriet Beecher Stowe, devenant ainsi le premier acteur noir à tenir l'un des premiers rôles dans un film américain destiné à un public blanc, les rôles de Noirs étant tenus jusque là par des acteurs blancs grimés - dans le film, Sam Lucas donne d'ailleurs la réplique à des comédiens blancs noircis au charbon.

42. L'American Folk Blues Festival est un festival itinérant qui va contribuer à populariser dans les années soixante le blues auprès de la jeunesse blanche européenne.

43. Ce qui n'empêchera pas les Rolling Stones d'apposer leur signature - Nanker Phelge, alors la signature collective du groupe avant que les compositions ne soient signées Jagger/Richards sur une composition de Bo Diddley, «I'm All Right».

44. "When you think of some dopey, spotty seventeen year old from Dartford, you know, who wants to be Muddy Waters-and there was a lot of us-in a way, very pathetic but in another way, very... heartwarming ". Interview de Keith Richards pour le documentaire de la BBC4 Can Blue Men Play The Whites, http://www.youtube.com/watch?v=-7B9tPuIP-w (page consultée le 15 octobre 2013).

45. Je rejoins donc Lhamon, au moins sur ce point précis, lorsqu'il affirme que « la question n'est

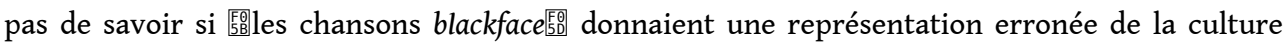


afro-américaine. On pourrait débattre à l'infini de la question de savoir si les ménestrels ont bien ou mal copié la culture noire. Mais on se heurterait toujours aux mêmes questions : qu'est-ce qu'une culture noire authentique? Y a-t-il quoi que ce soit d'authentique? Que veut dire “noir"?» (Lhamon, 2008, 75). Ainsi, la vraie question n'est pas tant celle de l'essence de la culture "noire » que celle de la logique à l'œuvre derrière la récupération du blues et du rhythm'n'blues.

\section{RÉSUMÉS}

Le spectacle de ménestrel blackface désigne une forme de théâtre populaire apparue dans la première moitié $d u x x^{e}$ siècle aux États-Unis qui voyait des comédiens, d'abord exclusivement Blancs, se noircir le visage à l'aide d'un bouchon de liège brûlé. Si, dans les années 1950, le «visage noirci » disparaît, la réalité que cette pratique recouvrait perdure. En me fondant sur une analyse de la musique rock, de ses origines et de ses circulations transatlantiques, j'examinerai les politiques raciales de cette musique en postulant une continuité du cycle blackface. Je souhaite ainsi montrer que l'exploitation commerciale qui a été faite de la musique noire a davantage intégré certains signes noirs plus que les Noirs eux-mêmes. Il sera ainsi question des performances de race à l'œuvre lorsque des artistes blancs comme Elvis Presley ou Mick Jagger ont porté un masque blackface, «empruntant » des signes à la tradition africaineaméricaine. Mais nous verrons aussi que des artistes noir-e-s « redécouvert-e-s » dans les années 1960 se sont également grimé-e-s pour servir à leur public blanc le frisson d'une supposée authenticité raciale.

Blackface minstrelsy is a form of popular American theater that gained popularity in the first half of the 19th century. As a form of theatrical makeup, blackface consisted of mostly white performers using burnt cork to blacken their skin. If the blackface makeup practice faded away in the 1950s, the reality behind it has carried on since. On the basis of an analysis of rock music, its origins and its transatlantic circulations, I will examine the racial politics of popular music and postulate a continuity of the blackface cycle. I intend to show that what has been integrated with the commercialization of black music is black culture more than Black people themselves. Thus, White artists, such as Elvis Presley or Mick Jagger, have worn a metaphorical blackface mask while "borrowing" signs from the African-American tradition. But some Black artists who were "rediscovered" in the 1960s (Blues Revival) also had to entertain their White audience with the thrill of a so-called "racial authenticity."

\section{INDEX}

Keywords : Race, blues, rock, blackface, Elvis Presley, blackness

Thèmes : La boîte à musique

Mots-clés : Race, blues, rock, blackface, Elvis Presley, blackness 
AUTEUR

KEIVAN DJAVADZADEH

Université Paris 8 